Itinerarios culturales y rutas turístico-culturales 12

\title{
Ruta de Don Quijote. Un personaje literario... un producto turístico
}

María Lourdes Campos Romero. Profesora titular de Análisis Geográfico Regional.

Universidad de Castilla-La Mancha

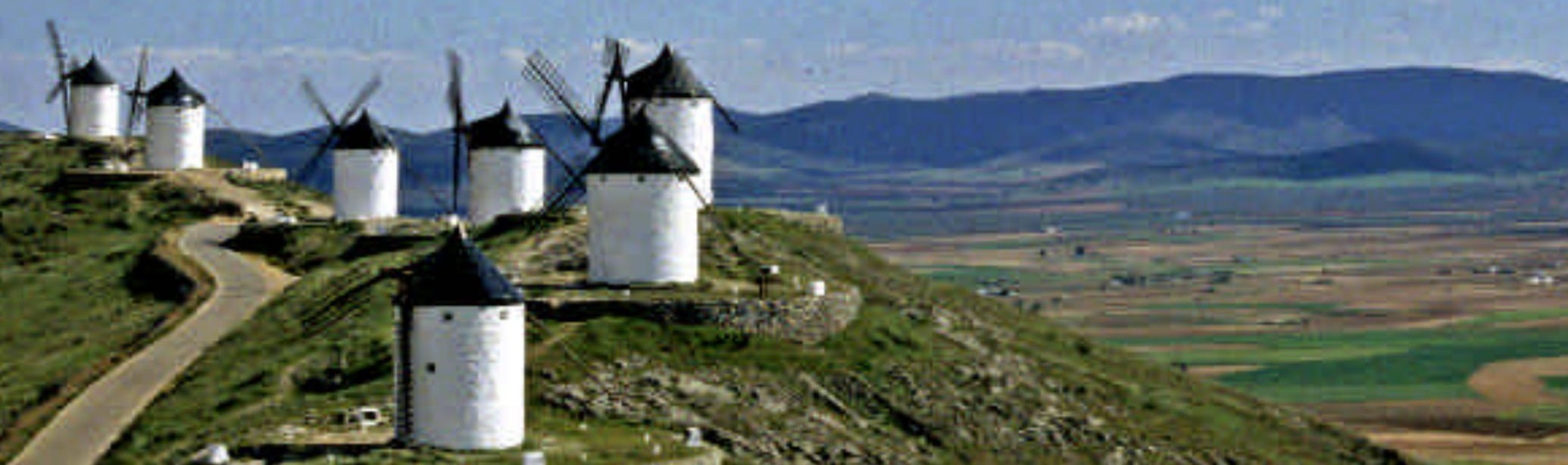


Con el objeto de conmemorar en 2005 el IV Centenario de la primera edición de la inmortal obra de Miguel de Cervantes El Ingenioso Hidalgo Don Quijote de la Mancha, la Junta de Comunidades de Castilla-La Mancha, entre otras actuaciones, adoptó la iniciativa de diseñar la Ruta de Don Quijote. Con ella pretendía dar a conocer la región y potenciar el turismo de interior, crear una red eco-turística con vocación de permanencia futura y generar la creación de riqueza. Intentaba aprovechar los recursos patrimoniales y paisajísticos de cada zona, poniendo en valor de manera integral todos los elementos destacables, tanto desde el punto de vista turístico como literario.

Por sus características, la Ruta de Don Quijote nace con vocación de transformarse en uno de los itinerarios eco-turísticos y culturales más extensos e importantes de España y de Europa.

\section{PROGRAMACIÓN, DISEÑO Y ELABORACIÓN}

El proyecto fue redactado en tan sólo tres meses, de febrero a mayo de 2004 , bajo la dirección técnica de GICAMAN por encargo de la Junta de Comunidades, a través de la empresa pública "Don Quijote de la Mancha 2005 ". La empresa pública TRAGSA preparó y redactó el proyecto de la Ruta de Don Quijote, cuyo diseño inicial se corres-

Q De los trece molinos originales que coronaban el Cerro Calderica (Consuegra, Toledo), en la actualidad sólo se conservan once, destacando entre ellos el denominado "Sancho", que aún posee toda la maquinaria del siglo XVI en óptimo estado para su funcionamiento / IsABEL BARRAGÁN ponde con un estudio promovido en el año 2000 por la Dirección General de Turismo de Castilla-La Mancha sobre rutas eco-turísticas preexistentes, conocido como "Proyecto Rocinante". También se encargó de ejecutar la obra sobre el terreno en un plazo de nueve meses. El 4 de mayo de 2004 el proyecto se entregó a la Consejería de Medio Ambiente para la supervisión técnica. Sobre el trazado propuesto inicialmente de 1.000 kilómetros, los técnicos de GICAMAN procedieron a un estudio de viabilidad que obligó a modificar el itinerario para obtener un trazado continuo de toda la Ruta que transcurriera por terrenos de titularidad pública, en función de las posibles afecciones medioambientales detectadas. El 31 de diciembre de 2004 la totalidad de la ejecución de las obras estuvo prácticamente terminada.

\section{ÁMBITO TERRITORIAL Y CONTENIDO PATRIMONIAL}

La Ruta de Don Quijote se extiende a lo largo de 2.386 kilómetros sobre 146 municipios de las provincias de Albacete, Ciudad Real, Cuenca, Guadalajara y Toledo. Se encuentra articulada por diez tramos diferenciados y éstos, a su vez, divididos en cincuenta y seis etapas, siendo el más extenso el primero de inicio de la Ruta, de Toledo a San Clemente, con 501 kilómetros y diez etapas y el más corto el noveno, de tan sólo 28 kilómetros y una etapa, que discurre por el norte de la provincia de Toledo en la zona de la Sagra.

Su recorrido vertebra el corazón de la Mancha y vincula la geografía regional a las andanzas de Don Quijote. La recuperación patrimonial de los caminos históricos que unen los municipios por donde discurre la Ruta, en ocasiones a punto de perderse por falta de uso, ha dado lugar a un proyecto presidido por la voluntad de proteger y favorecer el medio ambiente y la red caminera histórica. En esta 


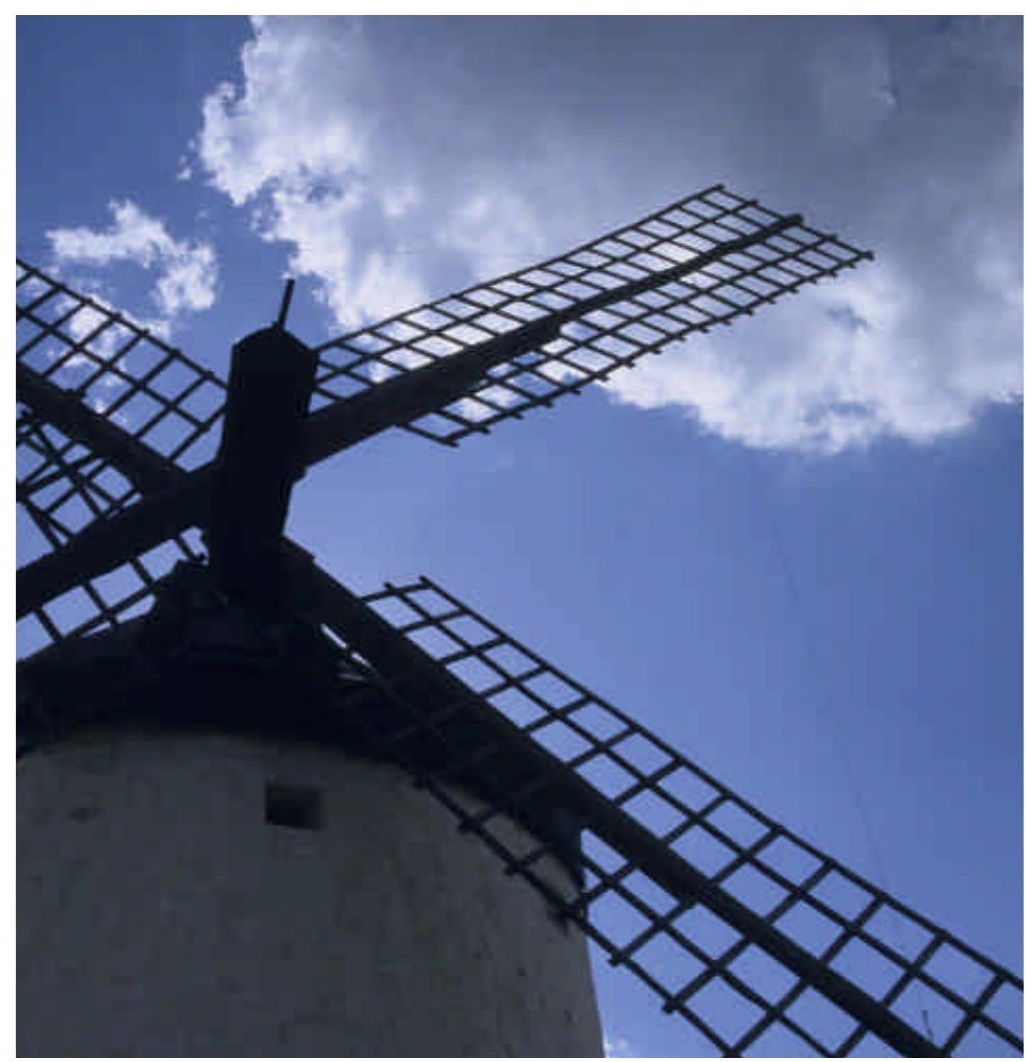

W Detalle de uno de los molinos de viento de Consuegra (Toledo), en lo alto del Cerro Calderico / Isabel Dugo

\section{Este proyecto une, mediante caminos rurales, las principales zonas de interés ecológico de La Mancha}

línea, se proyectó la recuperación de cañadas reales, cordeles, veredas, sendas y otras vías, así como la reforestación de algunos de los tramos y de las áreas de descanso incluidas en la Ruta, aparcamientos y descansaderos. Este proyecto une, mediante caminos rurales, las principales zonas de interés ecológico de La Mancha, entre las que se incluyen los más de 40 espacios protegidos, ya sean Lugares de Interés Comunitario (LIC), Zonas de Especial Protección para las Aves (ZEPA) o Espacios Naturales Protegidos (ENP). Este corredor verde es de uso prioritariamente peatonal, aunque también se puede recorrer en bicicleta y a caballo, e incluso algunas partes se pueden transitar en coche, estando concebido para unir núcleos urbanos y espacios naturales de Castilla-La Mancha.

Una de las particularidades de la Ruta es que todo su trazado discurre sobre ejes de dominio público lineal como vías pecuarias, caminos históricos, riberas fluviales y plataformas ferroviarias en desuso, aprovechando en buena parte los itinerarios de eco-corredores ya programados en el "Proyecto Rocinante". Además, es el primer itinerario de estas características que presenta hasta un $70 \%$ de su trayecto con accesibi lidad para personas discapacitadas. 


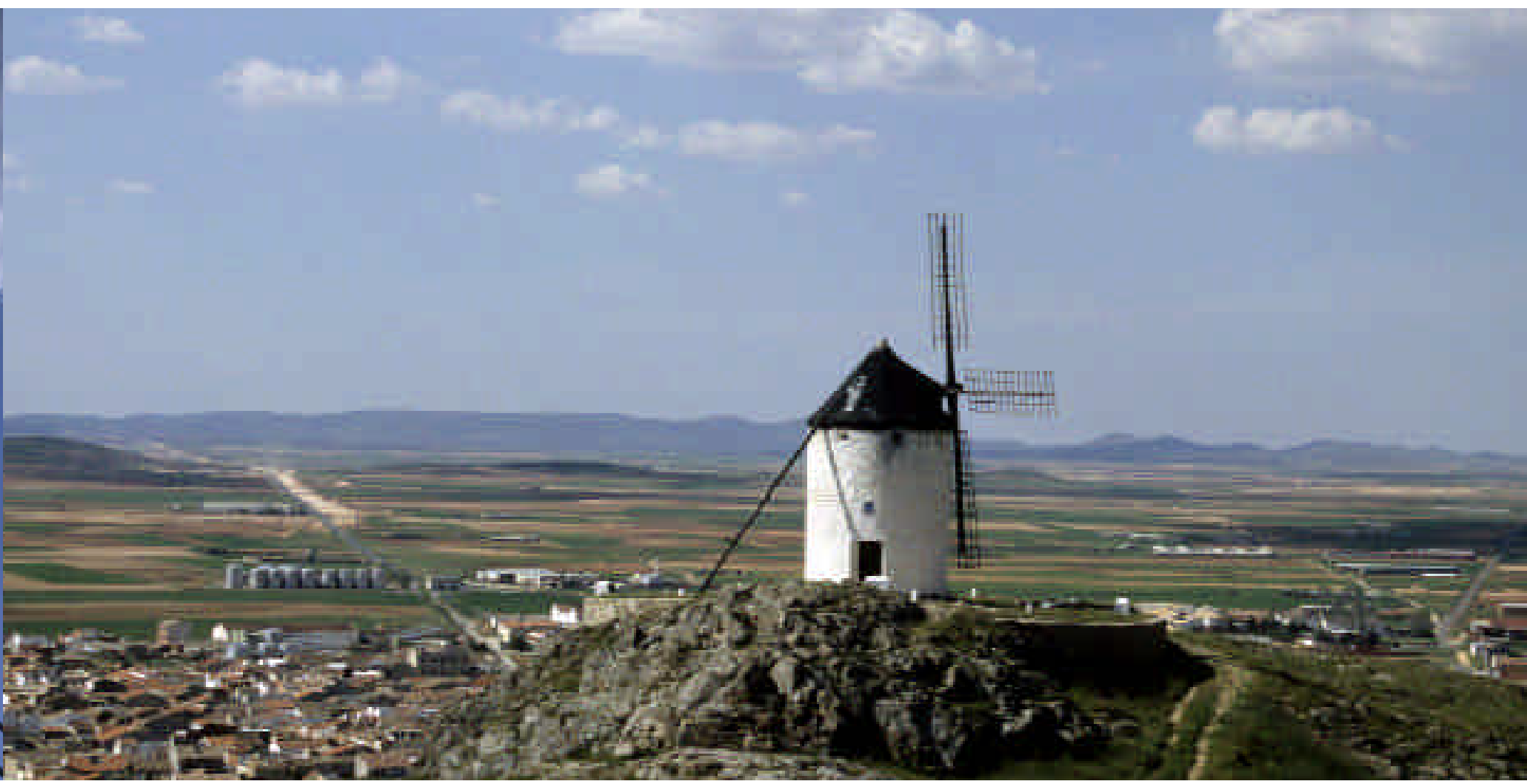

W Algunos de los molinos rehabilitados de Consuegra han sido rebautizados con nombres cervantinos: Mambrino, Rucio, Clavileño, Cardeño, Sancho... / Isabel Dugo

j Entidades participantes, promotoras y colaboradoras

Promueve la Junta de Comunidades de Castilla-La Mancha, a través de la Empresa Pública creada para tal fin, "Don Quijote de la Mancha 2005". A su vez, intervinieron en su elaboración y ejecución las Empresas Públicas "Gestión de Infraestructuras, S.A." (GICAMAN) y "Transformaciones Agrarias, S.A." (TRAGSA). Colaboran el Ministerio de Fomento, las Diputaciones Provinciales, los Ayuntamientos por los que discurre la Ruta y las Confederaciones Hidrográficas afectadas. Asimismo, RENFE, cofradías, hermandades y algunos particulares.

\section{j Financiación}

Toda la financiación es pública y procede íntegramente de la Junta de Comunidades de Castilla-La Mancha. La inversión presupuestada alcanza un total de 40 millones de euros, de los que 33 se destinan a la ejecución del proyecto y los 7 restantes al mantenimiento, conservación y reparaciones necesarias.

\section{j Estrategias patrimoniales}

Con la Ruta de Don Quijote se pretende recuperar y poner en valor un importante patrimonio natural e histórico artístico infrautilizado, en desuso y con riesgo de perderse. Con tal fin se ha procedido a la recuperación y restauración de elementos patrimoniales, religiosos y civiles, que jalonan la Ruta, y a la revalorización de los parques arqueológicos como elementos de dina mización cultural y turística.

\section{j Estrategias turísticas}

La recuperación de su vasto patrimonio permitirá convertir a Castilla-La Mancha en importante destino de turismo de interior, como lo evidencian los datos aportados sobre la afluencia de visitantes a distintas poblaciones y lugares de la Ruta. Consuegra en 2005 incrementó su turismo en un 70\%, en relación al año 2004, Campo de Criptana en un 50\% y Villanueva de los Infantes en un 400\%, lo que la convierte en estrella de la Ruta de Don Quijote. Igualmente, destaca la creación indirecta de nuevas casas rurales, con un $16 \%$ de incremento.

\section{j. De recurso a producto}

La Ruta fue presentada por primera vez en FITUR 2004 como Proyecto y promocionada durante 2005 en las principales ferias de turismo internacionales en coordinación con la Dirección General de Turismo y Turespaña. Asimismo, apareció publicitada en todos los medios de comunicación, con la imagen corporativa del IV Centenario, en 4.000 marquesinas y columnas y en 450 paneles informativos y de interpretación a todo lo largo de la Ruta, publicándose miles de folletos informativos sobre los diferentes tramos.

La administración autónoma trabaja en dos singulares distinciones para la Ruta, una de ellas ante la UNESCO, para su declaración como "Patrimonio Oral e Inmaterial de la Humanidad" a la figura y el legado de Don Quijote -incluida la Ruta-, y otra ante el Consejo de Europa para su declaración como "Itinerario Cultural Europeo", siendo la primera vez que se solicita la Declaración de Itinerario Cultural Europeo, referido a un personaje literario. 